\title{
Comparing dietary determinants of serum vitamin $D$ status among African-Americans and Caucasians
}

\author{
Sameer Murali, Lenore Arab ${ }^{*}$, Alfonso Ang, Casey Nelson, Katherine Wessling-Perry, \\ Patricia Jardack, Ronald L. Horst
}

Division of General Internal Medicine, David Geffen School of Medicine at UCLA, Los Angeles, USA

Email: larab@mednet.ucla.edu

Received 2 November 2011; revised 6 December 2011; accepted 15 December 2011

\begin{abstract}
African-Americans (AAs) maintain a high prevalence of vitamin D insufficiency compared to Caucasians. The relationship between dietary sources of vitamin $D$ and serum 25-hydroxyvitamin D (25(OH)D) among AAs remains poorly understood. This research examines the dietary determinants of 25(OH)D levels in AAs and Caucasians, controlling for demographic characteristics. 121 Caucasians and 119 AAs participated in a biomarker based study, comparing the correlation of serum 25(OH) D with dietary sources of vitamin D. Diet was assessed by multiple 24-hour (24hr) recall and Food Frequency Questionnaire (FFQ). Parallel multiple linear and logistic regression models stratified by race were then used to regress $25(\mathrm{OH}) \mathrm{D}$ level and sufficiency $(25(\mathrm{OH}) \mathrm{D}>20 \mathrm{ng} / \mathrm{mL})$ on major dietary sources of vitamin D-multivitamins (MV), milk, and fish-controlling for age, gender, and BMI. Results show that AAs are sensitive to the method of dietary assessment, with multiple $24 \mathrm{hr}$ recall demonstrating highest correlation (0.4) with $25(\mathrm{OH}) \mathrm{D}$ levels. MV's association with 25(OH)D levels was highest in both groups, $25 \%$ higher in AAs vs. Caucasians (8.6 vs. $6.5 \mathrm{ng} / \mathrm{mL}$ per $400 \mathrm{IU}$ ). Milk's impact on serum was similar in both groups but fish was only significant among Caucasians. MV consumption significantly increased odds of vitamin D sufficiency in both groups. Given the high prevalence of vitamin D insufficiency and the strong contribution as compared to milk or fish, MV supplementation is recommended. Additionally, future studies aimed at measuring vitamin D intake should consider the use of multiple $24 \mathrm{hr}$ recall instead of a single measure of FFQ or $24 \mathrm{hr}$ recall since the multiple $24 \mathrm{hr}$ recalls have a stronger correlation to serum $25(\mathrm{OH}) \mathrm{D}$.
\end{abstract}

Keywords: Vitamin D; Dietary Determinants; Fish; Vitamin Supplements

\footnotetext{
*Corresponding author.
}

\section{INTRODUCTION}

Race is often correlated with socioeconomic factors that differentially impact health outcomes, but rarely is skin color considered as an independent determinant of health. The inverse relationship between serum vitamin $\mathrm{D}$ $(25(\mathrm{OH}) \mathrm{D})$ status and skin pigmentation represents an exceptional case where physiology is driven by skin color alone [1]. Ninety-seven percent of African-American (AA) adults and adolescents are vitamin $\mathrm{D}$ insufficient $(<20$ $\mathrm{ng} / \mathrm{mL})$ or deficient $(<12 \mathrm{ng} / \mathrm{mL})$ [2]. Apart from the widespread prevalence of hypovitaminosis D among AAs, there are several reasons to study the determinants of 25 $(\mathrm{OH}) \mathrm{D}$ among AAs in particular.

First, AAs demonstrate unique physiologic handling of vitamin D. For example, compared to age-matched Caucasians, AAs demonstrate an adaptive response to vitamin D insufficiency that protects them from bone-related morbidity such as osteomalacia or osteoporotic fractures at low levels of vitamin D [3-5].

Second, while vitamin D is critical to bone health, its receptors act as transcription factors or cell-cycle mediators in nearly thirty tissues, suggesting a broader functional significance [6-8]. Many observational studies contend vitamin D insufficiency may play a role in the development of extra-skeletal illnesses such as cancer, diabetes, hypertension, and cardiovascular disease, all of which disproportionately affect AAs compared to Caucasians [9-15]. Experimental evidence supporting this remains mixed, and several randomized controlled trials are ongoing to define vitamin D's role in these conditions [16-20].

Third, existing evidence relating dietary intake to vitamin D status is often limited due to imprecise dietary assessment methods, such as single 24-hr recall or Food Frequency Questionnaires (FFQ) [21-25]. Single 24-hr dietary recalls cannot account for intra-individual dietary variations that occur over time. Despite this, they are routinely used in population surveys such as the National Health and Nutrition Examination Survey (NHANES). While FFQ are cheap and relatively easy to administer, 
validation studies using biomarkers find subjects significantly underreport caloric and protein intake [26]. FFQ have been used in cohort studies such as the Nurses Health Study, the Health Professionals Study, Coronary Artery Risk Development in Young Adults Study, and the Framingham Study.

African-Americans suffer from a disproportionately large prevalence of vitamin D insufficiency, which may be linked to many chronic illnesses. Supporting ongoing investigations into the impact of vitamin D supplementation on extra-skeletal morbidity in this vulnerable population requires a better understanding of the dietary determinants of vitamin D status. Thus far, limitations in dietary assessment have hampered our ability to grasp population-specific differences in dietary determinants of vitamin D status. This study attempts to address this by com- paring the association of dietary factors, assessed by single 24-hr recalls, FFQ, and multiple 24-hr recalls on serum vitamin D, among AAs and Caucasian subjects.

\section{METHODS}

\subsection{Data Source}

The UCLA Energetics Study was conducted in Los Angeles, California, between August 2006 and April 2009 [27]. The primary objective of the study was to validate a novel web-based, self-administered, 24-hr recall dietary assessment tool, known as DietDay, by comparing reported dietary intake with FFQ, as well as several biomarkers, including serum vitamin D. The UCLA Institutional Review Board (IRB) approved the study (IRB \# 11-001607), and all participants provided signed informed consent.

\subsection{Study Sample}

The UCLA Energetics Study recruited a convenience sample of generally healthy subjects through Craigslist.com, community notices, and posters distributed throughout the greater Los Angeles area. Those interested were referred to the study website where an automated self-screener determined their eligibility [28].

Eligibility was based on prospective participants' responses to questions regarding health status, age, ethnicity, smoking status, dietary, metabolic, and weight stability during the prior year. For the purposes of maintaining power for subgroup analyses, eligibility was restricted to ages 21 to 69, and to those who self-identified as "NonHispanic African-American" or "Non-Hispanic Caucasian."

Potential subjects were excluded due to any of the following conditions: gastrointestinal surgery, intestinal disease, pancreatic disease, diabetes, hemophilia, alcoholism, mental disorder, hypothyroidism, bipolar or seizure disorders congestive heart failure, renal failure or other conditions affecting fluid balance, current treatment with supplemental oxygen, antiretroviral, anti-neoplastic, anti-ulcer/anti-reflux, or central nervous system drugs. Smokers were excluded, as were respondents who could not endorse dietary, metabolic, and weight stability. Exclusion criteria based on health and smoking status as well as dietary, metabolic, and weight stability were developed to optimize the comparability of the biomarkers being measured between subjects.

Three hundred and thirty-three persons consented, 268 were scheduled into the study, 263 participants (99\% of those consented) completed the validation protocol involving multiple methods of dietary assessment and doubly labeled water (DLW) assays, and 243 subjects (91\%) had their serum vitamin D assays completed. We examined the data for outliers by examining the residuals. Observations with standardized residuals greater than \pm 3 were excluded from the analysis since these few extreme data points may exert undue influence on the regression results. Sensitivity analysis was also performed to examine the data with and without the outlying observations. After excluding outliers, the final sample for this analysis contained 119 African-Americans and 121 Caucasians. During the consent process, subjects received a detailed explanation regarding the DLW biomarker procedures, as well as instructions on how to access and complete the DietDay web-based 24-hour recalls.

\subsection{Outcome Measures}

Serum 25(OH)D was used to assess the vitamin D status of the study population. Concentration of 25(OH)D was determined by radioimmunoassay by Heartland Assays, Inc., Ames IA.

Vitamin D insufficiency and deficiency were defined based on the definitions established in the 2011 Institute of Medicine (IOM) Report on Dietary Reference Intakes for Calcium and Vitamin D. The IOM report defines vitamin $\mathrm{D}$ insufficiency as concentrations between 12 and $20 \mathrm{ng} / \mathrm{mL}$ and deficiency as a concentration $<12 \mathrm{ng} / \mathrm{mL}$.

\subsection{Variables}

The UCLA Energetics Study collected dietary information from subjects using different assessment methods. Each participant completed up to 8 self-administered online 24-hr dietary recalls (DietDay, Centrax Corporation, Chicago, IL), as well as the Diet History Questionnaire (DHQ), a food frequency questionnaire developed by the National Cancer Institute.

For the purposes of this analysis, we utilized the DietDay and DHQ assessed dietary intakes. The 1st DietDay 24-hr recall was completed at the consent visit. The 2nd DietDay was completed at the first clinic visit. The 3rd, 4th and 5th DietDays were scheduled on non-consecutive 
days between the first and second clinic visit, which was scheduled 8 to 14 days after the first visit. The 6th DietDay 24-hr recall was completed at the second clinic visit, where phlebotomy was also performed. Participants were scheduled to complete a 7th and 8th DietDay scheduled one month and two months after their first clinic visit, respectively. The day of week for DietDays was generated by an algorithm on the study website to ensure even distribution of the days of week reported for dietary assessment. An automated system notified subjects by e-mail to conduct the last two recalls without prior notice so that eating behavior would not be influenced. Subjects were e-mailed at $3 \mathrm{AM}$ and allowed until midnight of the same day to respond. If DietDays were not completed in a timely manner, the study coordinator followed up with a personalized e-mail or phone call.

DietDay contains 9349 foods and $>7000$ food images in 61 modules. Portion sizes are quantified by household measures using images of different amounts of food on a standard plate, glass, or bowl. Food preparation methods are also assessed, as well as condiments and additions. DietDay asks about usual consumption by time of day (midnight to 11 AM, 11 AM to 5 PM, and 5 PM to midnight). DietDay applies automatic branching, complex skip routines, range checks, edit checks, and prompts during the questionnaire. Nutrient values in the program were based on US Department of Agriculture values and expanded to include mixed dishes and product labeling information. DietDay was validated in both the AAs and Caucasian population in the context of the Energetics Study by estimating total energy expenditure from doubly labeled water assays and comparing them to reported energy intakes from multiple DietDay 24-hr recalls. The Energetics Study found DietDay to perform equally well if not better at assessing energy intake among AAs than Caucasians, and that it was feasible to receive as many as eight DietDay recalls from volunteers, without the perception of being burdensome [27,29].

The food sources included in the analyses were selected based on ranking foods among all DietDay-assessed 24-hr recalls by vitamin D content. The items that represented a majority of vitamin D consumed, including dairy items, fish, cold cereal, and multivitamin supplements, were selected for inclusion in the analyses. Dairy was further broken down into milk and cream. Milk was then subcategorized into skim, low-fat, and whole milk, and fish was subcategorized into oily fish and non-oily fish. Later subcategories were reconsolidated into milk and fish as the subcategories were insignificant on preliminary analyses. Total dietary calcium was also initially included, but later dropped due to its high correlation with milk consumption; cream and cold cereal were dropped do to their insignificant contribution to serum vitamin D levels based on initial analyses. Multivitamin supplements, fish, and milk consumption composed the final list of food source variables that were selected to include in the analyses.

In addition to diet, non-dietary factors also play a role in determining serum vitamin $\mathrm{D}$, such as age, gender, and body mass index (BMI). These elements were assessed and collected at the first study visit and subsequently included in our analyses as covariates.

\subsection{Statistical Analysis}

Analyses of 25(OH) vitamin D levels were completed using Stata/IC 11.0 for Mac and executed on the Mac OS X 10.5.8 platform.

AA and Caucasian participants were compared on age, gender, BMI, and education level using the chi-squared test. Mean total dietary intakes based on the multiple 24hr recall dietary assessment method for each group were compared using the Wilcoxon rank-sum test.

Comparing which dietary assessment method, FFQ, single 24-hr recall, or multiple 24-hr, recall best predicted $25(\mathrm{OH}) \mathrm{D}$ values was accomplished using two analytical methods: simple correlation and partial correlation. The partial correlation analysis controlled for age, gender, race, and BMI. Both 25(OH)D and total dietary vitamin D intake were log-transformed for the correlation analyses because scatter plots revealed a non-linear relationship between 25(OH)D and total dietary vitamin D. The correlations between AAs and Caucasians were tested using Fisher-z transformation test for differences between correlations.

Contribution of dietary and non-dietary characteristics to serum vitamin D was explored via ordinary least squares (OLS) multiple regression. In the regression analyses, serum and dietary vitamin D data were not log-transformed since the residuals from the regression did not violate the normality assumption. Dietary data for the OLS regression and the subsequent analyses were based on multiple 24-hr recalls. Two outliers were identified as having standardized residuals greater than 3 , and were excluded from all except for sensitivity analyses to determine their influence. The two outliers consist of one AA and one Caucasian, and after excluding these individuals, the results were very similar with the analysis that includes these outliers. None of the covariates changed more than $10 \%$ in value. Multiple regressions were stratified by race. In all the multivariate models, we controlled for potential confounders including age, gender, BMI and education. Power calculations show that this study had $80 \%$ power to detect a difference in natural log serum 25(OH)D between AAs and Caucasians for one standard deviation (0.50) difference between these race groups. This represents a change in serum 25(OH)D level of 13\% or more.

The associations of dietary and non-dietary determi- 
nants listed above on total dietary vitamin D intake were also analyzed via OLS regression and stratified by race. This was done to determine if total vitamin $\mathrm{D}$ dietary intake was differentially driven by a particular dietary source in either group, or if total intake was associated with specific non-dietary factors.

Multiple logistic regression analysis was also conducted to examine the association of dietary and non-dietary factors with vitamin D sufficiency status $(25(\mathrm{OH}) \mathrm{D}>$ $20 \mathrm{ng} / \mathrm{mL}$ ), stratified by race.

\section{RESULTS}

Population comparisons of AAs and Caucasians by age, gender, BMI, and education level are shown in Table $\mathbf{1 .}$ Both groups varied significantly from each other across these measures with the exception of gender.

Table 1. Population characteristics by race.

\begin{tabular}{|c|c|c|c|}
\hline & $\begin{array}{l}\text { African-American } \\
\mathrm{n}=\mathbf{1 1 9}\end{array}$ & $\begin{array}{l}\text { Caucasian } \\
\mathrm{n}=121\end{array}$ & p-values ${ }^{\mathrm{a}}$ \\
\hline Mean age (SD) ${ }^{\mathrm{b}}$ & $39(12)$ & $36(13)$ & 0.02 \\
\hline Gender (\%) & & & 0.06 \\
\hline Female & $83(70)$ & $70(58)$ & \\
\hline Male & $36(30)$ & $51(42)$ & \\
\hline Mean BMI (SD) ${ }^{\mathrm{b}}$ & $29(6.8)$ & $25(4.8)$ & $<0.01$ \\
\hline Education Level (\%) & & & $<0.01$ \\
\hline$<$ HS graduate & $1(1)$ & 0 & - \\
\hline HS graduate & $7(6)$ & 0 & - \\
\hline Some college & $60(50)$ & $32(26)$ & - \\
\hline College graduate & $43(36)$ & $61(50)$ & - \\
\hline Post graduate & $8(7)$ & $28(23)$ & - \\
\hline \multicolumn{4}{|c|}{ Mean dietary vitamin D intake IU (SD) ${ }^{\mathrm{b}, \mathrm{c}}$} \\
\hline Total & $188(203)$ & 207 (209) & 0.27 \\
\hline Multivitamin & $59(123)$ & $71(126)$ & 0.23 \\
\hline Milk, oz & $12(11)$ & $16(15)$ & 0.01 \\
\hline Fish, oz & $1(2)$ & $2(3)$ & 0.36 \\
\hline \multicolumn{4}{|c|}{ Serum vitamin D } \\
\hline $\begin{array}{l}\text { Mean 25(OH)D } \\
\text { ng/mL (SD) }\end{array}$ & $19(9)$ & $30(10)$ & $<0.01$ \\
\hline $\begin{array}{l}25(\mathrm{OH}) \mathrm{D}<20 \\
\mathrm{ng} / \mathrm{mL}(\%)\end{array}$ & $73(61)$ & $21(17)$ & $<0.01$ \\
\hline
\end{tabular}

${ }^{\mathrm{a}} \mathrm{p}$-values for age, BMI, dietary intake, and serum, all derived from Wilcoxon rank-sum test; gender and education level derived from chi-squared test; ${ }^{\mathrm{b}} \mathrm{SD}=$ standard deviation; ' Average intake as assessed by six $24 \mathrm{hr}$ dietary recalls.
Among African-Americans the mean serum 25(OH)D concentration was $19 \mathrm{ng} / \mathrm{mL}$. The prevalence of 25(OH)D insufficiency was 61\%. Among Caucasians the mean serum 25(OH)D concentration was $30 \mathrm{ng} / \mathrm{mL}$. The prevalence of $25(\mathrm{OH}) \mathrm{D}$ insufficiency was $17 \%$. Both groups demonstrated inadequate dietary intake of vitamin D based on multiple 24-hr recall data (188 IU vs. 207 IU, AAs vs. Caucasians respectively). The difference between the total intakes of both groups was not statistically significant; intake based on dietary source differed significantly only with respect to milk. Multivitamin supplementation contributed the single highest amount of dietary vitamin $\mathrm{D}$ in both racial groups.

Table 2 illustrates the correlation between reported dietary intake and 25(OH)D, as one compares FFQ to single 24-hr recall and single 24-hr recall to multiple 24-hr recalls by both simple and partial correlation methods. Multiple 24-hr recalls reflect the strongest correlative magnitude among all assessment methods. Their association with serum levels was greater among African Americans than Caucasians. Correlation coefficients between FFQ and 25(OH)D were 0.31 and 0.28 from simple and partial correlation methods respectively. Correlation strength was lower, at 0.27 and 0.22 based on a single 24-hr recall, but was highest at 0.30 and 0.41 based on the mean of six 24-hr recalls for simple and partial correlations respectively. Using Fisher-z transformation test, the overall partial correlation for 24-hr recall was significantly higher than the FFQ (0.36 vs. $0.20, p=0.03$ ). Based on the greater magnitude of correlation between multiple 24-hr recalls and serum levels, this dietary assessment method was retained for the multiple linear and logistic regressions.

There was no significant association between gender and $25(\mathrm{OH}) \mathrm{D}$ concentration in AAs, whereas among Caucasians, females averaged $4.6 \mathrm{ng} / \mathrm{mL}$ lower 25(OH)D concentration than males (Table 3). BMI was significantly associated with lower 25(OH)D concentration in both groups, although the magnitude was almost twice as great among Caucasians compared to African-Americans, 0.5 $\mathrm{ng} / \mathrm{mL}$ per unit of BMI versus $0.3 \mathrm{ng} / \mathrm{mL}$ per unit of BMI respectively. Education was not predictive of $25(\mathrm{OH}) \mathrm{D}$ concentrations for both AAs and Caucasians. We also performed sensitivity analysis on our final multivariate models by including physical activity as a possible covariate, this was, however, not significant.

With regard to dietary determinants, 400 IU multivitamin consumption contributed most to the estimation of serum levels in both groups. Among AAs, multivitamin consumption was associated with $8.6 \mathrm{ng} / \mathrm{mL}$ higher se rum levels per $400 \mathrm{IU}$ consumed, versus $6.4 \mathrm{ng} / \mathrm{mL}$ higher per 400 IU among Caucasians (Table 3). Milk consumption was significantly associated with higher serum levels similarly in both groups, $3.0 \mathrm{ng} / \mathrm{mL}$ per oz versus 3.7 
Table 2. Correlations between log of total dietary vitamin D (mcg) with log of serum 25(OH)D (ng/mL) by assessment method.

\begin{tabular}{|c|c|c|c|c|c|c|}
\hline & \multicolumn{3}{|c|}{ Simple Correlations } & \multicolumn{3}{|c|}{ Partial Correlations $^{\mathrm{a}}$} \\
\hline & All $n=240$ & $\mathrm{AAs}^{\mathrm{b}} \mathrm{n}=119$ & Caucasians n = 121 & All $n=240$ & $\mathrm{AAs}^{\mathrm{b}} \mathrm{n}=119$ & Caucasians $n=121$ \\
\hline $24 \mathrm{hr}$ recall $\times 6$ days & 0.32 & 0.4 & $0.31^{\mathrm{c}}$ & $0.36^{\mathrm{d}}$ & 0.36 & 0.41 \\
\hline 24 hr recall $\times 1$ day & 0.29 & 0.27 & 0.21 & 0.25 & 0.27 & 0.22 \\
\hline FFQ & 0.25 & 0.13 & $0.30^{\mathrm{c}}$ & $0.20^{\mathrm{d}}$ & 0.14 & 0.28 \\
\hline
\end{tabular}

${ }^{\mathrm{a}}$ Covariates include age, gender, race, BMI and education; ${ }^{\mathrm{b}} \mathrm{AAs}=$ African-Americans; ${ }^{\mathrm{c}}$ Significant differences between AA \& CAU at $\mathrm{p}=0.05$; ${ }^{\mathrm{d}}$ Significant difference between 24 HR \& FFQ, $p=0.03$.

Table 3. Multiple linear regression model results for serum 25(OH)D (ng/mL) on dietary sources stratified by race.

\begin{tabular}{|c|c|c|c|c|c|c|c|c|c|}
\hline & \multicolumn{3}{|c|}{ Overall sample } & \multicolumn{3}{|c|}{ African-Americans ${ }^{\mathrm{a}}$} & \multicolumn{3}{|c|}{ Caucasians $^{\mathrm{a}}$} \\
\hline & Coefficient & $\mathrm{SE}^{\mathrm{b}}$ & p-value & Coefficient & SE & p-value & Coefficient & SE & $\mathrm{p}$-value \\
\hline Multivitamin (per pill) & 7.39 & 1.95 & 0.001 & 8.6 & 2.4 & 0.001 & 6.44 & 2.5 & 0.01 \\
\hline Milk (per oz) & 3.41 & 1.09 & 0.002 & 3.02 & 1.35 & 0.03 & 3.67 & 1.4 & 0.01 \\
\hline Fish (per oz) & 1.53 & 0.71 & 0.05 & 1.31 & 1.02 & 0.25 & 1.91 & 0.8 & 0.02 \\
\hline Age (years) & -0.14 & 0.05 & 0.01 & -0.05 & 0.08 & 0.54 & -0.16 & 0.07 & 0.01 \\
\hline \multicolumn{10}{|l|}{ Males (ref) } \\
\hline Females & -2.74 & 1.31 & 0.04 & -1.71 & 2.04 & 0.4 & -4.55 & 1.57 & 0.01 \\
\hline BMI, kg/m² & -0.42 & 0.11 & 0.001 & -0.32 & 0.13 & 0.02 & -0.52 & 0.18 & 0.004 \\
\hline \multicolumn{10}{|l|}{$<$ College graduate (ref) } \\
\hline College graduate & -0.92 & 1.3 & 0.48 & -0.64 & 1.83 & 0.73 & -1.39 & 1.94 & 0.77 \\
\hline \multicolumn{10}{|l|}{ Caucasians (ref) } \\
\hline African-Americans & -7.28 & 1.37 & 0.001 & - & & & - & & \\
\hline
\end{tabular}
${ }^{\mathrm{a}}$ Total variance explained in the linear regression model, or adjusted R-squared, for African-Americans $=0.14$, Caucasian $=0.25$, overall total sample = 0.36;
${ }^{\mathrm{b}} \mathrm{SE}=$ standard error.

ng/mL per oz serving among African-Americans and Caucasians respectively. Fish consumption did demonstrate a significant association with higher serum 25(OH)D levels among African-Americans, but among Caucasians, fish consumption was significantly associated with 25(OH)D levels.

Odds of being 25(OH)D sufficient, or demonstrating serum levels $>20 \mathrm{ng} / \mathrm{mL}$, significantly decreased with age among Caucasians (OR = 0.95; 95\% CI, 0.90 - 0.99), but had no significant impact among African-Americans (Table 4). The effect of gender on sufficiency status differed greatly between the racial groups. Among Caucasians, females were less likely to have vitamin D sufficiency (OR $=0.07,95 \% \mathrm{CI}, 0.01-0.37$ ). Gender was not significant in determining 25(OH)D sufficiency status among AAs. Each unit increase in BMI significantly increased odds of insufficiency in both racial groups; Caucasians were almost 2 times that of African-Americans (13\% versus 8\%
Table 4. Multiple logistic regression model results for vitamin D sufficiency (>20 ng/mL) on dietary sources and demographic characteristics by race.

\begin{tabular}{ccccc}
\hline & $\begin{array}{c}\text { African-Americans } \\
\mathbf{n}=\mathbf{1 1 9}\end{array}$ & \multicolumn{2}{c}{ Caucasians $^{\mathrm{a}} \mathbf{\text { N = 121 }}$} \\
\hline & OR & CI & OR & CI \\
Multivitamin (400 IU) & 1.44 & $1.17-1.78$ & 1.35 & $1.02-1.78$ \\
Milk (per oz) & 1.06 & $1.02-1.67$ & 1.06 & $1.01-1.12$ \\
Fish (per oz) & 1.23 & $0.98-1.79$ & 1.3 & $1.01-1.67$ \\
Age (years) & 0.99 & $0.95-1.03$ & 0.95 & $0.90-0.99$ \\
Males (ref) & & & & \\
Females & 0.76 & $0.30-1.92$ & 0.07 & $0.01-0.37$ \\
BMI, kg/m & 0.92 & $0.86-0.99$ & 0.87 & $0.77-0.98$ \\
$<$ College Graduate (ref) & & & & \\
College Graduate & 0.67 & $0.27-1.66$ & 0.7 & $0.18-2.70$ \\
\hline
\end{tabular}

${ }^{\mathrm{a}}$ Pseudo R-square for AA = 0.15, Caucasians $=0.29$. 
respectively). Multivitamin consumption significantly increased odds of sufficiency similarly in both groups; 44\% among African-Americans and 38\% among Caucasians. Milk consumption significantly increased odds of sufficiency in African-Americans and among Caucasians. Fish consumption was significantly associated with sufficiency status in AAs but not among Caucasians.

\section{DISCUSSION}

This study is unique in that it examines the impact of dietary determinants of vitamin D status among AAs compared to Caucasians using a two stage approach: initially evaluating multiple dietary assessment methods to determine the best method for comparing dietary determinants among the two groups, then exploring the independent association of various dietary sources on serum status and vitamin D sufficiency.

Our findings indicate that multiple 24-hr dietary recalls maintained superior correlation with $25(\mathrm{OH}) \mathrm{D}$ compared to single 24-hr recall and FFQ among AAs since the overall partial correlation was significantly higher (Table 2). Among Caucasians, FFQ and multiple 24-hr dietary recalls outperformed single 24-hr recall. McAlindon et al. published a correlation coefficient of 0.24 between FFQassessed dietary vitamin D and 25(OH)D among participants of the Framingham Study, similar to our simple correlation of FFQ assessed dietary vitamin D and 25(OH) $\mathrm{D}$ for all participants of 0.25 [30].

Though several large population-based observational studies have described the relationship between dietary intake and vitamin D status, studies rarely report the correlation between assessed vitamin $\mathrm{D}$ intake and serum levels, and no study has compared multiple dietary assessment methods on the basis of correlational strength with $25(\mathrm{OH}) \mathrm{D}$. When combined with the known existing limitations of dietary assessment methods, this leaves the validity of any conclusions regarding the relationship of dietary vitamin D intake and $25(\mathrm{OH}) \mathrm{D}$ status up for debate [2,31-33]. Overall, our results suggest that dietary assessment can vary by racial group and future studies with racially diverse populations should carefully consider the method of dietary assessment employed.

Our study demonstrates that both AAs and Caucasians consumed similarly low quantities of vitamin D. Previous authors have described low dietary intake of vitamin D among both African Americans and Caucasians, but with median intake among AAs as 6\% - 31\% which is lower than those of Caucasians of comparable age and sex. This has partially been attributed to a higher prevalence of lactose intolerance among AAs compared to Caucasians, reducing AA consumption of vitamin D-rich dairy products $[33,34]$. Our data reveals a small but significant difference in milk consumption between AAs and Caucasians (2 IU versus 3 IU), but total dietary in- take of vitamin D was similar in both groups. Despite the similarity in dietary intake, serum levels were on average $36 \%$ lower among AAs than Caucasians at $19 \mathrm{ng} / \mathrm{mL}$ vs. $30 \mathrm{ng} / \mathrm{mL}$ respectively. The gap in serum levels found in our data compares somewhat favorably to a populationbased study by Ginde et al., which reported a 44\% disparity between AAs and Caucasians based on NHANES 2001-2004 data at $15 \mathrm{ng} / \mathrm{mL}$ and $27 \mathrm{ng} / \mathrm{mL}$ respectively.

Much of the disparity in serum levels could be attributable to lower vitamin D acquisition from UVB radiation due to darker skin pigmentation among AAs. If so, one may expect a compensatory mechanism of higher vitamin D acquisition from diet among AAs compared to Caucasians. However, when determining associations between dietary sources of vitamin D and 25(OH)D levels, we found a mixed pattern of associations in each racial group. While multivitamin consumption's association with 25(OH)D levels was 25\% higher in AAs vs. Caucasians, milk consumption impacted both groups similarly, and fish consumption only significantly impacted serum levels among Caucasians. These results are not entirely explained by differences in consumption patterns either, as consumption of each dietary source only differed significantly with respect to milk.

One possible way to reconcile this pattern is to consider the differences in non-dietary determinants with 25 (OH)D levels. Among Caucasians, all non-dietary determinants demonstrated significant associations with serum levels, in complete contrast with African-Americans. This suggests that while determinants of 25(OH)D levels in Caucasians are both dietary and non-dietary, among AAs, determinants are less multifaceted and largely dependent on dietary intake.

Examining the associations of dietary sources and nondietary factors with determination of vitamin D sufficiency as defined by the recent Institute of Medicine (IOM) report of $20 \mathrm{ng} / \mathrm{mL}$, confirms this assertion. The overall impact of dietary sources on serum 25(OH)D sufficiency was nearly identical in both groups. Multivitamin supplementation featured as the major dietary determinant of sufficiency associated with 35\% - 44\% higher odds of sufficiency, however among non-dietary factors, female gender was associated with 93\% lower odds of serum sufficiency compared to men among Caucasians, with no significant gender impact on sufficiency among AAs. Described another way, being male was associated with 14 fold higher odds of serum sufficiency among Caucasians, while gender showed no association with sufficiency among AAs. This analysis not only underscores the importance of vitamin supplementation in achieving serum sufficiency irrespective of race, but also demonstrates that non-dietary factors can eclipse dietary mediators among Caucasians, while dietary intake stands out as the primary determinant of status among AAs. 
This study has limitations as well as strengths. The sample population, although diverse in age, education and $\mathrm{BMI}$, is a select group of adult volunteers. The applicability of these findings to population based studies as well as to other age, geographical and ethnic groups will require validation. This analysis involves a small sample size and our inability to assess sun-exposure in the sample population. Seasonal differences present during collection of 25(OH)D were not accounted for. The crosssectional design further limits our ability to comment on the causal nature of the observed associations, and may overestimate the magnitude of actual findings. The study also did not assess serum calcium and parathyroid hormone. However, the strength of this study lies in the fact that two methods of assessment of vitamin D intake were used (24-hr recall and FFQ). The recruitment sample is well-balanced between AAs and Caucasians (119 vs. 121), allowing for reasonable comparisons to be made. Clinical lab data involving biomarkers were obtained from the participants including doubly-labeled water (DLW) and serum vitamin $\mathrm{D}$ the day after intake assessments were made (24-hr recall and FFQ).

In conclusion, diet plays an important role in determining vitamin D status among AAs. Regular multivitamin consumption containing 400 IU of vitamin D was associated with a $1 / 3$ greater likelihood of sufficiency. The impact on serum was much lower from other dietary sources of vitamin D in both groups. Given the large prevalence of vitamin D insufficiency and its association with negative health outcomes, vitamin D supplementation should be recommended among AAs. Additionally, future studies aimed at measuring dietary intake in AAs may consider FFQ and single 24-hr recall as inferior methods compared to multiple 24-hr recalls, which can be effectively implemented using self-administered, webbased platforms.

\section{ACKNOWLEDGEMENTS}

This study was supported in part by National Institutes of Health Grant 5R01CA105048-04 and UCLA, General Clinical Research Centers Program Grant M01-RR000865. The collection of data follows the procedures in accordance with the ethical standards of the institution and IRB approval was obtained for this study. The authors acknowledge the excellent support of Jasmine Yaxun Chen and Nidhi Shetty in the preparation of the manuscript. None of the authors had a conflict of interest.

\section{REFERENCES}

[1] Holick, M.F., MacLaughlin, J.A., Clark, M.B., et al. (1980) Photosynthesis of previtamin D3 in human skin and the physiologic consequences. Science, 210, 203-205. doi:10.1126/science.6251551

[2] Ginde, A.A., Liu, M.C. and Camargo, C.A. Jr. (2009)
Demographic differences and trends of vitamin D insufficiency in the US population, 1988-2004. Archives of Internal Medicine, 169, 626-632. doi:10.1001/archinternmed.2008.604

[3] Dawson-Hughes, B., Harris, S.S., Finneran, S. and Rasmussen, H.M. (1995) Calcium absorption responses to calcitriol in black and white premenopausal women. Journal of Clinical Endocrinology \& Metabolism, 80, 30683072. doi:10.1210/jc.80.10.3068

[4] Dawson-Hughes, B., Harris, S., Kramich, C., Dallal, G. and Rasmussen, H.M. (1993) Calcium retention and hormone levels in black and white women on high- and lowcalcium diets. Journal of Bone and Mineral Research, 8, 779-787. doi:10.1002/jbmr.5650080702

[5] Barrett, J.A., Baron, J.A., Karagas, M.R. and Beach, M.L. (1999) Fracture risk in the U.S. medicare population. Journal of Clinical Epidemiology, 52, 243-249. doi:10.1016/S0895-4356(98)00167-X

[6] Norman, A.W. (1998) Receptors for 1alpha,25(OH)2D3: Past, present, and future. Journal of Bone and Mineral Research, 13, 1360-1369. doi:10.1359/jbmr.1998.13.9.1360

[7] Nemere, I. and Farach-Carson, M.C. (1998) Membrane receptors for steroid hormones: A case for specific cell surface binding sites for vitamin D metabolites and estrogens. Biochemical and Biophysical Research Communications, 248, 443-449. doi:10.1006/bbrc.1998.8492

[8] Deluca, H.F. and Cantorna, M.T. (2001) Vitamin D: Its role and uses in immunology. The FASEB Journal, 15, 2579-2585. doi:10.1096/fj.01-0433rev

[9] Wang, T.J., Pencina, M.J., Booth, S.L., et al. (2008) Vitamin D deficiency and risk of cardiovascular disease. Circulation, 117, 503-511. doi:10.1161/CIRCULATIONAHA.107.706127

[10] Poole, K.E., Loveridge, N., Barker, P.J., et al. (2006) Reduced vitamin D in acute stroke. Stroke, 37, 243-245. doi:10.1161/01.STR.0000195184.24297.c1

[11] Mensah, G.A., Mokdad, A.H., Ford, E.S., Greenlund, K.J. and Croft, J.B. (2005) State of disparities in cardiovascular health in the United States. Circulation, 111, 12331241. doi:10.1161/01.CIR.0000158136.76824.04

[12] Melamed, M.L., Muntner, P., Michos, E.D., et al. (2008) Serum 25-hydroxyvitamin D levels and the prevalence of peripheral arterial disease: Results from NHANES 2001 to 2004. Arterioscler Thromb Vasc Biol, 28, 1179-1185. doi:10.1161/ATVBAHA.108.165886

[13] Forman, J.P., Giovannucci, E., Holmes, M.D., et al. (2007) Plasma 25-hydroxyvitamin D levels and risk of incident hypertension. Hypertension, 49, 1063-1069. doi:10.1161/HYPERTENSIONAHA.107.087288

[14] Fiscella, K. and Franks, P. (2010) Vitamin D, race, and cardiovascular mortality: Findings from a national US sample. Annals of Family Medicine, 8, 11-18. doi:10.1370/afm.1035

[15] Dobnig, H., Pilz, S., Scharnagl, H., et al. (2008) Independent association of low serum 25-hydroxyvitamin d and 1,25-dihydroxyvitamin d levels with all-cause and 
cardiovascular mortality. Archives of Internal Medicine, 168, 1340-1349. doi:10.1001/archinte.168.12.1340

[16] Effect of vitamin D3 supplementation on insulin resistance and cardiovascular risk factors in obese adolescents NCT00858247.

http://clinicaltrials.mayo.edu/clinicaltrialdetails.cfm?trial id $=101180$

[17] Study of vitamin D and effect on heart disease and insulin resistance NCT01093417. http://clinicaltrials.gov/ct2/show/NCT01093417

[18] Randomised, multi-centre, double-blind, placebo-controlled trial of vitamin D supplementation in adult and adolescent patients with asthma ISRCTN07270894. http://www.ukctg.nihr.ac.uk/trialdetails/ISRCTN0727089 $\underline{4}$

[19] A randomised double blind placebo controlled phase II multicentre pilot study to investigate the effects of vitamin D2 or D3 supplementation on metabolic parameters in people at risk of type 2 diabetes ISRCTN86515510. http://apps.who.int/trialsearch/trial.aspx?trialid=ISRCTN 86515510

[20] Can high-dose vitamin D supplementation reduce blood pressure and markers of cardiovascular risk in older people with isolated systolic hypertension? A randomised, double-blind, parallel group placebo controlled trial ISRCTN92186858.

http://www.controlled-trials.com/ISRCTN92186858

[21] Kristal, A.R., Peters, U. and Potter, J.D. (2005) Is it time to abandon the food frequency questionnaire? Cancer Epidemiology Biomarkers \& Prevention, 14, 2826-2828. doi:10.1158/1055-9965.EPI-12-ED1

[22] Kipnis, V., Subar, A.F., Midthune, D., et al. (2003) Structure of dietary measurement error: Results of the OPEN biomarker study. American Journal of Epidemiology, 158, 14-21.

[23] Jackson, K.A., Byrne, N.M., Magarey, A.M. and Hills, A.P. (2008) Minimizing random error in dietary intakes assessed by 24-h recall, in overweight and obese adults. European Journal of Clinical Nutrition, 62, 537-543. doi:10.1038/sj.ejcn.1602740

[24] Holmes, B., Dick, K. and Nelson, M. (2008) A comparison of four dietary assessment methods in materially deprived households in England. Public Health Nutrition, 11, 444-456. doi:10.1017/S1368980007000559

[25] Basiotis, P.P., Welsh, S.O., Cronin, F.J., Kelsay, J.L. and Mertz, W. (1987) Number of days of food intake records required to estimate individual and group nutrient intakes with defined confidence. Journal of Nutrition, 117, 16381641.

[26] Schatzkin, A., Kipnis, V., Carroll, R.J., et al. (2003) A comparison of a food frequency questionnaire with a 24-hour recall for use in an epidemiological cohort study: Results from the biomarker-based Observing Protein and Energy Nutrition (OPEN) study. International Journal of Epidemiology, 32, 1054-1062. doi:10.1093/ije/dyg264

[27] Arab, L., Wesseling-Perry, K., Jardack, P., Henry, J. and Winter, A. (2010) Eight self-administered 24-hour dietary recalls using the Internet are feasible in African Americans and Whites: The energetics study. Journal of the American Dietetic Association, 110, 857-864. doi:10.1016/j.jada.2010.03.024

[28] Arab, L., Hahn, H., Henry, J., Chacko, S., Winter, A. and Cambou, M.C. (2010) Using the web for recruitment, screen, tracking, data management, and quality control in a dietary assessment clinical validation trial. Contemporary Clinical Trials, 31, 138-146. doi:10.1016/j.cct.2009.11.005

[29] Arab, L., Tseng, C.H., Ang, A. and Jardack, P. (2011) Validity of a multi-pass, web-based 24-hour self administered recall for assessment of total energy intake as strong among African Americans as Caucasians. American Journal of Epidemiology, in press.

[30] McAlindon, T.E., Felson, D.T., Zhang, Y., et al. (1996) Relation of dietary intake and serum levels of vitamin D to progression of osteoarthritis of the knee among participants in the Framingham Study. Annals of Internal Medicine, 125, 353-359.

[31] Looker, A.C., Pfeiffer, C.M., Lacher, D.A., Schleicher, R.L., Picciano, M.F. and Yetley, E.A. (2008) Serum 25-hydroxyvitamin D status of the US population: 19881994 compared with 2000-2004. American Journal of Clinical Nutrition, 88, 1519-1527. doi:10.3945/ajcn.2008.26182

[32] Harris, S.S. (2006) Vitamin D and African Americans. Journal of Nutrition, 136, 1126-1129.

[33] Calvo, M.S., Whiting, S.J. and Barton, C.N. (2004) Vitamin D fortification in the United States and Canada: Current status and data needs. American Journal of Clinical Nutrition, 80, 1710S-1716S.

[34] Moore, C.E., Murphy, M.M. and Holick, M.F. (2005) Vitamin D intakes by children and adults in the United States differ among ethnic groups. Journal of Nutrition, 135, 2478-2485. 